\title{
Der Griff nach den Sternchen. Zum Umgang mit gegenderten Schreibweisen im Berliner Journal für Soziologie
}

Online publiziert: 19. November 2019

(C) Springer Fachmedien Wiesbaden GmbH, ein Teil von Springer Nature 2019

Die Soziologie ist eine heikle Wirklichkeitswissenschaft. Sie beschreibt und klassifiziert Akteure, die sich selbst klassifizieren und auf Klassifikationen durch andere reagieren, und sie bedient sich dabei sprachlicher Mittel, die voller sozialer und politischer Verweise stecken und Wertladungen aufweisen. Insofern ist die Soziologie in ihrer Bezeichnungspraxis zugleich machtvoll. Jede sozialwissenschaftliche Kategorisierung ist nicht einfach nur ein Abbild von Realität, sondern eine Auswahl unter Bezeichnungs- und Ordnungsmöglichkeiten, also problematisierbar, manchmal sogar problematisch. Die Soziologie macht Individuen zu Dividuen und ordnet sie Kollektiva zu, selbst wenn diese als sichtbare und aktive Gruppen womöglich gar nicht in Erscheinung treten. Das gilt auch für die Kategorisierung von Geschlechtern.

Auch wenn stets darauf geachtet wurde, dass die binären Geschlechterbezeichnungen im Berliner Journal nicht diskriminierend gebraucht werden, wurde und wird von den Autorinnen und Autoren immer wieder der Wunsch vorgebracht, in ihren Texten eine strikt gendergerechte Schreibweise verwenden zu dürfen. Darunter wird in der Regel eine Schreibweise verstanden, bei der bei jeder generalisierenden Akteursbezeichnung, die sich auf natürliche Personen bezieht, alle Geschlechter sprachlich adäquat repräsentiert werden, und zwar durch ein um ein Binnenzeichen ergänztes hybrides Begriffskompositum anstelle der nur binären Geschlechterbezeichnungen. Die zentralen Argumentationsmuster, die dieses Anliegen stützen, sind bekannt: Die häufig noch verbreitete ausschließliche Verwendung des generischen Maskulinums - was ja auch eine Form des Genderns ist - kann als Unsichtbarmachen von Frauen und anderen Geschlechtern verstanden, als Diskriminierung interpretiert werden oder Repräsentationsansprüche verletzen. Sprache bildet nicht nur Realität $\mathrm{ab}$, sondern schafft sie auch, indem sie unser Denken beeinflusst und damit zur Wirklichkeitskonstruktion und zur Errichtung von spezifischen Wissenssystemen z. B. in Form von stereotypen Rollen- und Geschlechterbildern beiträgt.

Die Frage der Umsetzung des Anspruchs auf einen gendergerechten Sprachgebrauch in den jeweiligen sozialen Kontexten und Wissensfeldern ist damit allerdings 
noch nicht beantwortet. Die Soziologie in ihren vielfältigen Verzweigungen und theoretischen Zugängen macht zwangsläufig einen exzessiven Gebrauch von Kategorisierungen von Personen und Klassenkollektiva, die sie teils aus der allgemeinen Sprache übernimmt, teils selbst (re-)konstruiert oder erfindet, teils umcodiert oder formalisiert. Zudem ist das Deutsche hochgradig genusgebunden, ruft also ständig Geschlechterassoziationen hervor, selbst da, wo sie sachlich nicht unbedingt bestehen. Nicht die Verwendung von gendergerechten Personen- oder Gruppenbezeichnungen ist jedoch das Kernproblem, auch wenn sie Fragen der konsistenten Anwendbarkeit eines gewählten Schemas und ggf. ästhetische Fragen aufwirft. Deutlich schwerer wiegt die Tatsache, dass die Soziologie - wie andere Wissenschaften auch - eine komplexe Fachsprache mit einer komplizierten Syntax und Grammatik pflegt, die diesen Personenkategorien zwangsläufig Pronomen, bestimmte und unbestimmte Artikel, Adjektive sowie Rück- und Querverweise zuordnet, die ebenfalls genusadäquat gebeugt werden müssen. Eine grammatikalisch konsequente genderalternierende bzw. genderoffene Schreibweise kann so zu kaum noch lesbaren Wortund Satzkonstruktionen führen.

Gleichwohl haben der Herausgeberkreis und die Redaktion des BJS beschlossen, das Journal mit Beginn des Jahrgangs 2019 für gängige alternative Genderschreibweisen zu öffnen, falls die Autorinnen und Autoren das wünschen bzw. für geboten halten. Voraussetzung für die Verwendung alternativer Darstellungsformen ist, dass die gewählte Genderschreibweise fachsprachlichen Standards genügt, konsistent angewandt wird, die grammatikalischen Folgeprobleme bewältigt werden sowie die Lesbarkeit des Textes erhalten bleibt und nachvollziehbar ist, welche Funktion der Schreibweise im jeweiligen Text zukommt.

Eine praktikable und gut lesbare Variante, die die Intention einer möglichst inklusiven Genderschreibweise wahrt, die alternierenden Beugungen der Artikel, Adjektive und Pronomen aber vermeidet, kann aus unserer Sicht darin bestehen, dass diese nur auf die letzten Endungen der jeweiligen Personenklassentermini hin gebeugt werden, auf die sie sich beziehen - also in der Regel auf das generische Femininum hin, weil das meist am Ende steht. Dann hieße es etwa: Eine problembewusste Lehrer_in, der die Freizeit ihrer Schüler_innen am Herzen liegt, sollte den Einsatz von Hausaufgaben genau abwägen (statt: Ein_e problembewusste_r Lehrer_in, dem/der die Freizeit seiner/ihrer Schüler_innen am Herzen liegt, sollte...; analog sind die Varianten mit Schrägstrich, Binnen-I oder Sternchen möglich). Diese genderoffene, aber sparsame Schreib- und Beugungsform löst nicht alle grammatikalischen Probleme, sie stellt aus unserer Sicht aber einen praktikablen Kompromiss dar, der zu flüssig lesbaren Texten führt. Darüber hinaus steht es den Autorinnen und Autoren selbstverständlich frei, die bislang im BJS geläufigen Genderschreibweisen weiter $\mathrm{zu}$ verwenden. ${ }^{1}$

\footnotetext{
$1 \mathrm{Zu}$ diesen Varianten gehören neben dem generischen Maskulinum die ausgeschriebene Genus-Doppelung, der Genus-Tausch (nur das generische Femininum) und die stochastische Genus-Wahl, jeweils bei Bedarf mit Fußnote zur Erläuterung der gewählten Variante und einem Hinweis auf die Inklusion aller Geschlechter. Ausführlichere Informationen zu möglichen Genderschreibweisen im BJS mit einem längeren Beispielsatz, der die von uns vorgeschlagene Variante und die zu meisternden Komplikationen etwas ausführlicher erläutert, finden sich in Kürze auf der Verlagsseite des BJS.
} 
Ob sich diese Regelung auch im praktischen Redaktionsalltag bewährt und wo ggf. weitere Fallstricke lauern, ist eine offene Frage. Das wird die Zusammenarbeit mit den Autorinnen und Autoren und das Lektorat der Texte zeigen. Zudem werden wir die Reaktionen und Hinweise unserer Leserinnen und Leser berücksichtigen. Wir behalten uns deshalb Nachjustierungen bei der Regelung der Genderschreibweisen vor, falls sich dies als sinnvoll oder geboten erweisen sollte. Zudem ist nicht auszuschließen, dass in näherer Zukunft durch die Dudenredaktion doch noch ein Regelkanon entwickelt wird, der auf alle Textsorten anwendbar ist.

Welche praktischen Implikationen die vorgeschlagene Regelung hat, lässt sich im vorliegenden Heft den Beiträgen von Koppetsch et al., Knopp und Ullrich sowie Aunkofer et al. entnehmen. Wie der letztgenannte, von Stefanie Aunkofer, Christine Wimbauer, Benjamin Neumann, Michael Meuser und Katja Sabisch verfasste und auf einer qualitativen Studie beruhende Text zeigt, können mit der Nutzung des * durchaus Präzisionsgewinne verbunden sein: verhandeln hier doch Partner*innen über die Elternzeit der Partner, die anders als die der Partnerinnen noch immer die Ausnahme ist. Und erst, wenn als Untersuchungseinheit das Paar in seiner inneren Dynamik in den Blick genommen wird, offenbart sich, wie die Autor*innen zeigen, die Bandbreite der im Paar verhandelten Orientierungsrahmen und Deutungsmuster für die väterliche Elternzeitnahme.

Um Paare dreht sich auch der Beitrag von Cornelia Koppetsch, Eva-Maria Bub und Judith Eckert - allerdings um solche, die als Ex-Partner*innen wieder getrennte Wege gehen. Hier geht es um eine Frage, die ebenso alltäglich wie existenziell ist und geradezu sinnbildlich für den Einbruch des Realen ins menschliche Imaginäre steht: warum Beziehungen scheitern. Auch in dieser qualitativen Studie wird auf verschiedene Konstellationen verwiesen. Hinterfragt werden emotionale Reziprozitätserwartungen und Machtkonstellationen in Paaren, die sich vor allem im Ungleichgewicht von Geben und Nehmen zeigen. Die Autorinnen entdecken so Machtasymmetrien am Werk, die es in sich haben: Wider Erwarten ist es im Regelfall nämlich nicht die stärkere, sondern die schwächere Partner*in, die der Beziehung ein Ende setzt, weil sie weniger abhängig von ihr ist als ihr Gegenüber. Und das ist in dieser Studie relativ unabhängig vom konkreten Geschlecht.

Dass nicht nur Lebens-, sondern auch Geschäftspartner*innen aneinander vorbeireden können, zeigt eindrücklich der Beitrag von Philip Neeb und Sebastian Knake, der sich der Beziehung zwischen Banken und ihren störrischen Kund*innen widmet. Gegenstand der Analyse ist die wechselseitige Intransparenz von Erwartungen zwischen Banken und deren Kundschaft, die sich aus der Sicht der Banken als Rationalitätsproblem und aus der Sicht der Kundschaft als Vertrauensproblem deuten lässt. Dies wird von den Autoren mit Rückgriff auf Studien aus dem Zeitraum zwischen 1959 und 1992, die aus der Perspektive der Banken durchgeführt wurden, sowie auf der Grundlage eigener aktueller Analysen aus Kund*innensicht detailliert gezeigt und theoretisch interpretiert.

Philipp Knopp und Peter Ullrich befassen sich in ihrem Beitrag mit Macht- und Subjektivierungseffekten der Videoüberwachung auf Demonstrationen. Theoretisch knüpft der Beitrag an den Diskurs um Macht- und Regierungstechniken an und erweitert diesen um das Konzept der Subjektivierungsweisen. Auch hier geht es em- 
pirisch darum, verschiedene Deutungsmuster herauszuarbeiten. Diese auf Basis von Gruppendiskussionen mit Teilnehmer*innen verschiedener politischer Richtungen (von rechten bis linksradikalen Gruppen) und von Ultra-Fußballfans identifizierten Wahrnehmungen, Deutungen und Handlungsstrategien zeigen, dass Videoüberwachung seitens der Polizei nicht nur der Abschreckung, Überwachung und Kontrolle dient und als solche interpretiert wird. Die unterschiedlichen Protestkulturen deuten abhängig von ihren Staats- und Polizeibildern und der jeweiligen Protestsituation die Videoüberwachung sehr verschieden und entwickeln entsprechend auch unterschiedliche Reaktionsweisen.

Thomas Schwinn setzt sich in seinem Essay kritisch mit Hans Joas’ Buch „Die Macht des Heiligen“ auseinander. Die Kritik setzt dabei v. a. an Joas' Lesart von Max Webers These der „Entzauberung“ und „Rationalisierung“ der Welt an. Vor diesem Hintergrund diskutiert Schwinn Joas' Verständnis des Verhältnisses von Transzendenz und Immanenz und argumentiert, dass Transzendenz bei Joas anthropologisch interpretiert wird, Selbsttranszendenz zwar thematisiert, aber nicht auf Lebensführungs- und Ordnungseffekte befragt wird, und so Verweltlichungsprozesse nicht erklärt werden können. Schwinn versteht Joas als einen Säkularisierungskritiker, der aber eben nicht soziologisch, sondern eher anthropologisch argumentiert.

Schließlich diskutiert Nico Sonntag in seiner Forschungsnotiz neuere Versuche, quantitative netzwerkanalytische Verfahren für die Erforschung der Ideengeschichte des ökonomischen Denkens und des Neoliberalismus einzusetzen. Am Beispiel einer neueren Untersuchung zu Netzwerken deutscher Nachkriegsökonomen (die in diesem Fall tatsächlich männlich dominiert waren) setzt sich der Autor kritisch mit den dabei angewendeten Methoden auseinander und entwickelt eine eigene Ideenskizze, die sowohl konzeptionelle Ansatzpunkte aufzeigt wie auch Fragen der Stichprobenziehung, der Operationalisierung von „Kanten“ und „Knoten“ sowie der Datenaufbereitung thematisiert.

Der Blick auf die Beiträge in diesem Band zeigt die Vielfalt der Möglichkeiten im Umgang mit sprachlichen Bezeichnungen von diversen Genusgruppen - die natürlich den unterschiedlichen Präferenzen der Autor*innen folgen, zugleich aber auch den Erfordernissen des jeweiligen Gegenstands und seiner wirklichkeitswissenschaftlichen Durchdringung geschuldet sind. Dem Griff nach den (soziologischen) Sternen eines besseren Verständnisses gesellschaftlicher Realitäten ist und bleibt das Berliner Journal verpflichtet. Wo es dieser Sache dient, kann dabei von nun an auch auf das Sternchen - oder auf Unterstrich, Schrägstrich oder Binnen-I zurückgegriffen werden.

\section{Die Herausgeber*innen und die Redaktion}

